\title{
The roles of eotaxin and the STAT6 signalling pathway in eosinophil recruitment and host resistance to the nematodes Nippostrongylus brasiliensis and Heligmosomoides bakeri
}

\author{
M.L. Knott ${ }^{\mathrm{a}}$, K.I. Matthaei ${ }^{\mathrm{b}, \mathrm{c}}$, P.S. Foster ${ }^{\mathrm{b}, \mathrm{d}}$, L.A. Dent ${ }^{\mathrm{a}, *}$ \\ a School of Molecular and Biomedical Science, University of Adelaide, Adelaide, SA 5005, Australia \\ ${ }^{\mathrm{b}}$ John Curtin School of Medical Research, Australian National University, Canberra, ACT 0200, Australia \\ c Department of Anatomy, College of Medicine and King Khalid University Hospital, King Saud University, Riyadh 11461, Saudi Arabia \\ d School of Biomedical Sciences, University of Newcastle, Newcastle, NSW 2300, Australia
}

\section{A R T I C L E I N F O}

\section{Article history:}

Received 16 April 2009

Accepted 14 May 2009

Available online 16 June 2009

\section{Keywords:}

STAT6

IL-4R $\alpha$

IL-13

Eotaxin

Nippostrongylus brasiliensis

Primary and secondary resistance

\begin{abstract}
A B S T R A C T
Expulsion of adult Nippostrongylus brasiliensis worms from the small intestine is profoundly impaired in signal transducer and activator of transcription (STAT)6-deficient mice. IL-5 transgenic (Tg) mice with constitutive eosinophilia show profound early resistance in the skin and/or later pre-lung phase of primary infections with $N$. brasiliensis. This study was designed to assess the importance of the eosinophil chemokine eotaxin and the STAT6/interleukin (IL)-4/IL-13 signalling pathway in early resistance to $N$. brasiliensis. Eosinophil recruitment into the skin following injection of $N$. brasiliensis larvae was reduced in STAT6- or eotaxin-deficient/IL-5 Tg double mutant mice. While ablation of eotaxin did not impair resistance in the pre-lung phase of $N$. brasiliensis infections in IL-5 Tg mice, elimination of STAT6 caused a modest reduction in resistance in both primary and secondary infections on this genetic background. STAT6 $^{-1-}$-, IL-13-/- - and IL-4R $\alpha^{-1-}$-deficient single mutant and IL-13-1- /L-4R $\alpha^{-1-}$ double mutant mice were more susceptible than WT mice during the pre-lung phase of secondary $N$. brasiliensis infections. In contrast, primary or secondary resistance were unaffected at either the pre-lung or gut stages of infection in eotaxin ${ }^{-1-}$ single mutant mice. STAT6 ${ }^{-1-}$ and eotaxin ${ }^{-1-}$ mice with or without the IL-5 transgene, were no more susceptible than WT or IL-5 Tg mice to protracted primary infections with Heligmosomoides bakeri, a parasitic nematode that is restricted to the gut. Our data suggest that parasitic nematodes that transit through the skin and lungs en route to the gut may be susceptible to early (pre-lung) innate and adaptive immune mechanisms that are dependent on the STAT6/IL-4/IL-13 signalling pathway, and this may be important for the development of effective therapies and vaccines.
\end{abstract}

(c) 2009 Elsevier Ltd. All rights reserved.

\section{Introduction}

Eosinophils are typically involved in allergies, asthma and parasitic helminth infections and in most instances, their roles are controversial and not well understood. Eosinophil numbers are often elevated in the peripheral blood and in tissues infected by parasitic helminths. Complement is important for the very early recruitment of eosinophils, when larvae first enter the skin, but other factors begin to mediate eosinophil recruitment within the first $3 \mathrm{~h}$ of infection (Giacomin et al., 2008). In experimental models of asthma, the recruitment of eosinophils into the lungs is largely dependent on the chemokine eotaxin, which has a high degree of specificity for this leukocyte subset (Collins et al., 1995; Gonzalo et al., 1996; Mattoli et al., 1997). Eotaxin was first identified in

\footnotetext{
* Corresponding author. Tel.: +61 88303 4155; fax: +61 883034362

E-mail address: lindsay.dent@adelaide.edu.au (L.A. Dent).
}

guinea pig lungs after aerosol challenge with ovalbumin, and was found to induce eosinophil accumulation in the skin (Jose et al., 1994). Eotaxins are constitutively expressed in a range of tissues, including the lungs, gut and some lymphoid tissues (Matthews et al., 1998; Zimmermann et al., 2000) and can be induced via the IL-4/IL-13/signal transducer and activator of transcription (STAT)6 signalling pathway (Zimmermann et al., 2003), in airway smooth muscle cells (Moore et al., 2002) and human fibroblasts (Mochizuki et al., 1998). Induction of eotaxin expression by IL-4 and/or IL-13 and subsequent recruitment of eosinophils has been associated with allergen-induced pathology in the lungs and large intestine (Kweon et al., 2000). In eotaxin-deficient mice, basal tissue eosinophil levels are reduced (Mould et al., 1997), allergen-induced recruitment of eosinophils into the lungs is impaired (Rothenberg et al., 1997) and these mice are less susceptible to gastrointestinal allergy (Hogan et al., 2000).

The intestinal phase of Nippostrongylus brasiliensis infections has been intensively studied to determine the factors and pathways 
required for expulsion of adult worms (Lawrence et al., 1996; Urban et al., 1998, 2001). Less attention has been given to host resistance mechanisms that might operate in other tissues. Eosinophils are recruited to the jejunum after $N$. brasiliensis larvae arrive in the lumen (Dent et al., 1999) and there is some evidence to suggest that these leukocytes may provide protection in the intestinal phase of primary infections (Knott et al., 2007). However, the skin is the site of entry for a number of helminthic parasites of humans and our previous work suggests that innate immune mechanisms can be protective from the early skin and pre-lung stages of $N$. brasiliensis infections. More broadly, the pre-lung phase of infection may represent a promising avenue for the prevention of some helminth infections (Daly et al., 1999; Knott et al., 2007; Giacomin et al., 2008).

The IL-4/IL-13/STAT6 signalling pathway is necessary for expulsion of worms from the small intestine and IL-4R $\alpha$ and STAT6 in particular, have been shown to be most important (Urban et al., 1998). This pathway may in part provide protection against $N$. brasiliensis via the induction of eotaxin and subsequent recruitment of eosinophils to the gut, but possibly also in earlier stages of the infection. In the present study, we investigated the kinetics of infections with $N$. brasiliensis and with another intestinal nematode, Heligmosomoides bakeri, in mice genetically deficient in either eotaxin-1, STAT6, IL-13 or the IL-4 receptor (R) $\alpha$, or both IL-13 and the IL- $4 R \alpha$, as well as mice over expressing an IL- 5 transgene but lacking STAT6 or eotaxin. Cellular immune responses were assessed during both primary and secondary nematode infections. For $N$. brasiliensis infections, particular attention was given to the early skin response and larval migration to the lungs.

\section{Materials and methods}

\subsection{Animals}

Heterozygous male and female BALB/c mice with approximately 49 copies of the IL- 5 transgene (derived by greater than 20 backcrosses from CBA/Ca line Tg5C2, abbreviated IL-5 Tg (Dent et al., 1990)) and their wildtype (WT) littermates were bred in the Medical School Animal House of the University of Adelaide. BALB/c eotaxin-1-deficient (eotaxin-1-l-) (Mishra et al., 1999) and STAT6deficient $\left(\right.$ STAT6 $^{-1-}$ ) (Kaplan et al., 1996) mice either with or without the IL-5 transgene, IL-13-deficient (IL-13-l-) (McKenzie et al., 1998), IL-4 R $\alpha$-deficient (IL-4R $\alpha^{-l-}$ ) (Noben-Trauth et al., 1997) and IL-13/IL-4R $\alpha$ double-deficient (IL-13-/- IL-4R $\alpha^{-/-}$) mice were bred at The John Curtain School of Medical Research, Australian National University. All mice were closely age matched within experiments. Hooded Wistar rats used for passage of $N$. brasiliensis were obtained from Laboratory Animal Services at the University of Adelaide. All animals were handled according to the guidelines of the University of Adelaide Animal Ethics Committee.

\subsection{Parasitological techniques}

\subsubsection{Parasite maintenance}

Infective $N$. brasiliensis third stage larvae (L3) were obtained by the faecal culture method after passage through Hooded Wistar rats as described previously (Daly, 1999), washed twice in MilliQ water and once in mouse osmolality phosphate buffered saline (MPBS) (Sheridan and Finlay-Jones, 1977). L3 were injected subcutaneously (s.c.) at the base of the neck (750L3/mouse) or into air pouches (500L3/mouse) in the mid-dorsal skin using a 19-gauge needle. Mice in secondary infection groups were injected s.c. with $750 \mathrm{~L} 3$ for lung and intestinal analyses or $500 \mathrm{~L} 3$ for air pouch experiments and 3 weeks later, after clearance of the primary infection, mice were challenged with 750 or 500 L3 respectively.
Infective $H$. bakeri (previously known as $H$. polygyrus; Cable et al., 2006) L3 were also obtained by the faecal culture method, but passage was performed in $\mathrm{BALB} / \mathrm{c}$ mice using oral gavage of infective L3. Larvae were stored at $4{ }^{\circ} \mathrm{C}$ until used, and each experimental animal received $200 \mathrm{~L} 3$ by oral gavage.

\subsubsection{Parasite recovery from tissues}

$N$. brasiliensis lung larvae (L4) were recovered from animals on day 2 post infection (p.i.) as previously described (Knott et al., 2007). Faecal egg counts (6-27 days p.i. for $N$. brasiliensis and at various time points from 13 to 116 days p.i. for $H$. bakeri) and intestinal worm burdens (day 7 p.i. for $N$. brasiliensis and days 41 and 117 p.i. for $H$. bakeri) were also performed as previously described (Giacomin et al., 2005; Knott et al., 2007).

\subsection{Generation of skin air pouches}

Air pouches were created in the shaved dorsal skin of anaesthetised mice, as described previously (Daly et al., 1999; Giacomin et al., 2008). Briefly, $2.5 \mathrm{ml}$ of filtered air was subcutaneously injected to inflate the skin. Three days later, pouches were reinflated with $2 \mathrm{ml}$ of sterile air and after a further 3 days, $500 \mathrm{~L} 3$ were injected/mouse. Air pouches were lavaged 3-4 times with $2 \mathrm{ml}$ of MPBS, 0 and $4 \mathrm{~h}$ after injection of larvae. The viable cells and larvae recovered were enumerated and differential leukocyte counts were estimated from cytocentrifuged samples, with a minimum of 200 cells counted per sample using oil immersion light microscopy.

\subsection{Histology}

Lungs were removed on day 2 p.i. and the post-caval lobe (right lung) was taken for histology. Small intestines were removed on day 7 p.i. and as previously described (Dent et al., 1999) a $1 \mathrm{~cm}$ piece from the duodenum was taken for sectioning. Tissues were fixed in $10 \%$ buffered formalin and embedded in paraffin. Five $\mu \mathrm{m}$ sections were cut and stained with haematoxylin \& eosin (H \& E), counted and/or photographed at $20 \times$ objective magnification.

\subsection{Statistical analysis}

Parasite burdens and inflammatory leukocyte numbers were compared using the non-parametric Kruskal-Wallis (KW) test, followed by Mann-Whitney (MW) $U$ post hoc testing or parametric two-way ANOVA followed by Bonferroni post hoc testing in Prism Version 5.01 (GraphPad Software Inc., La Jolla, CA, USA). In all tests $P<0.05$ was considered significant.

\section{Results}

\subsection{Larval retention and leukocyte recruitment in skin air pouches}

Larvae were injected into dorsal skin air pouches and primary and secondary inflammatory responses were assessed in WT, IL-5

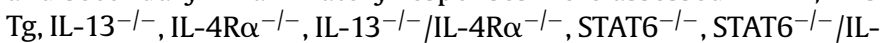
$5 \mathrm{Tg}$, eotaxin-1-/- and eotaxin-1-/-/IL-5 Tg animals 0 and $4 \mathrm{~h}$ after infection. Larvae were recovered from the air pouch lumen, however, at $4 \mathrm{~h}$ p.i. there were no significant differences between groups and $6-42 \%$ of the inoculum was recovered per group (data not shown). As previously reported (Daly et al., 1999), the total number of cells recovered from each group at $0 \mathrm{~h}$ was minimal (mean $<2 \times 10^{6}$ cells/mouse). Total cell numbers recovered from air pouches at $4 \mathrm{~h}$ p.i. in secondary infections of mice carrying the IL-5 transgene were similar to or slightly greater than those seen during a primary infection $\left(2-6 \times 10^{6}\right.$ total leukocytes/mouse). In 
both primary and secondary infections, similar total pouch leukocyte numbers were recovered from mice deficient in IL-13, IL-4R $\alpha$, STAT6, eotaxin- 1 and WT mice (1.8-4.3 $\times 10^{6}$ total leukocytes). The total number of neutrophils did not vary greatly either between primary and secondary infections within strains, or between strains (1-4 $\times 10^{6}$ total neutrophils).

As expected, eosinophils were recruited in large numbers $4 \mathrm{~h}$ p.i. in IL-5 Tg mice in both primary and secondary infections (Fig. 1) and there was a significant effect between strains. Higher numbers of eosinophils were recovered from air pouches of mice carrying the IL- 5 transgene than in other groups in both primary and secondary infections, but this was reduced if eotaxin-1 was absent (Fig. 1A). Relative to primary infections, a significant increase in eosinophil recruitment occurred in secondary infections in the absence of either IL-4R $\alpha$ or IL-13, but not when both were deleted (Fig. 1B). Further, during secondary infections, eosinophil numbers were reduced in IL-5 Tg mice lacking STAT6 (Fig. 1C). Lymphocytes and macrophages were not a prominent feature of the inflammatory infiltrate and no notable differences were seen between groups (data not shown).

\subsection{Lung larval recovery and cellular response}

To further investigate the potential importance of eotaxin-1, IL-13, IL-4 and STAT6 in resistance to primary and secondary $N$. brasiliensis infections, we assessed the capacity of larvae to migrate to the lungs by day 2 p.i. (Fig. 2) and the inflammatory infiltrate induced (Fig. 3). Lung larval numbers typically peak by day 2 p.i. in primary infections of WT mice. Within a host type, there were significant reductions in lung larval burdens in primary vs. secondary infections for all lines except IL-4R $\alpha^{-1-}$ and IL-13-/- IL-4R $\alpha^{-1-}$ (Fig. 2A-C). During secondary infections of IL-13-1-, IL-4R $\alpha^{-1-}$, IL-13 ${ }^{-1-} /$ IL-4R $\alpha^{-1-}$ and STAT6 ${ }^{-1-}$ mice, significant numbers of larvae reached the lungs, i.e. unlike in infections in WT mice, larvae were not trapped in the pre-lung stage of infection (Fig. 2B and C). Eotaxin-1-l- mice had similar lung larval burdens to WT mice in both primary and secondary infections (Fig. 2A). During primary and secondary infections of eotaxin-1 ${ }^{-1-} /$ IL-5 Tg mice, larval numbers were low and at comparable levels to those found in IL$5 \mathrm{Tg}$ mice (Fig. $2 \mathrm{~A}$ ). In both primary and secondary infections, STAT6 $^{-/-} /$IL-5 Tg mice had slight but significantly increased numbers of lung larvae, relative to those detected in single mutant IL-5 Tg controls, but were still substantially more resistant than WT mice (Fig. 2C). All groups had a modest cellular inflammatory response in the lungs at day 2 p.i., with few eosinophils present (Fig. 3 and not shown). As previously reported (Knott et al., 2007), expression of the IL-5 transgene did not substantially increase cell recruitment to the lungs at this stage of either primary or secondary infections (data not shown). Relative to WT (Fig. 3A and B) mice, an absence of eotaxin (Fig. 3C and D) or STAT6 (Fig. 3E and F) did not impair cell recruitment in either primary or secondary infections. IL-13-/-, IL-4R $\alpha^{-1-}$ and IL-13-/- /IL-4R $\alpha^{-1-}$ mice had similar lung histology to STAT6 $^{-1-}$ mice (data not shown).

\subsection{Intestinal phase of infection}

While much work has been done to investigate the roles of IL-13, IL-4 and STAT6 during the intestinal phase of primary $N$. brasiliensis infections (Urban et al., 1998; Madden et al., 2002, 2004; Sakamoto et al., 2004), little has been published on the significance of this pathway during secondary infections. Further, the role of eotaxin in recruiting eosinophils to the gut during $N$. brasiliensis infections has not been characterised. In primary infections of many WT mouse strains, larvae and worms typically localise in the anterior end of the small intestine from 3 to 9 days p.i. and eggs are produced from
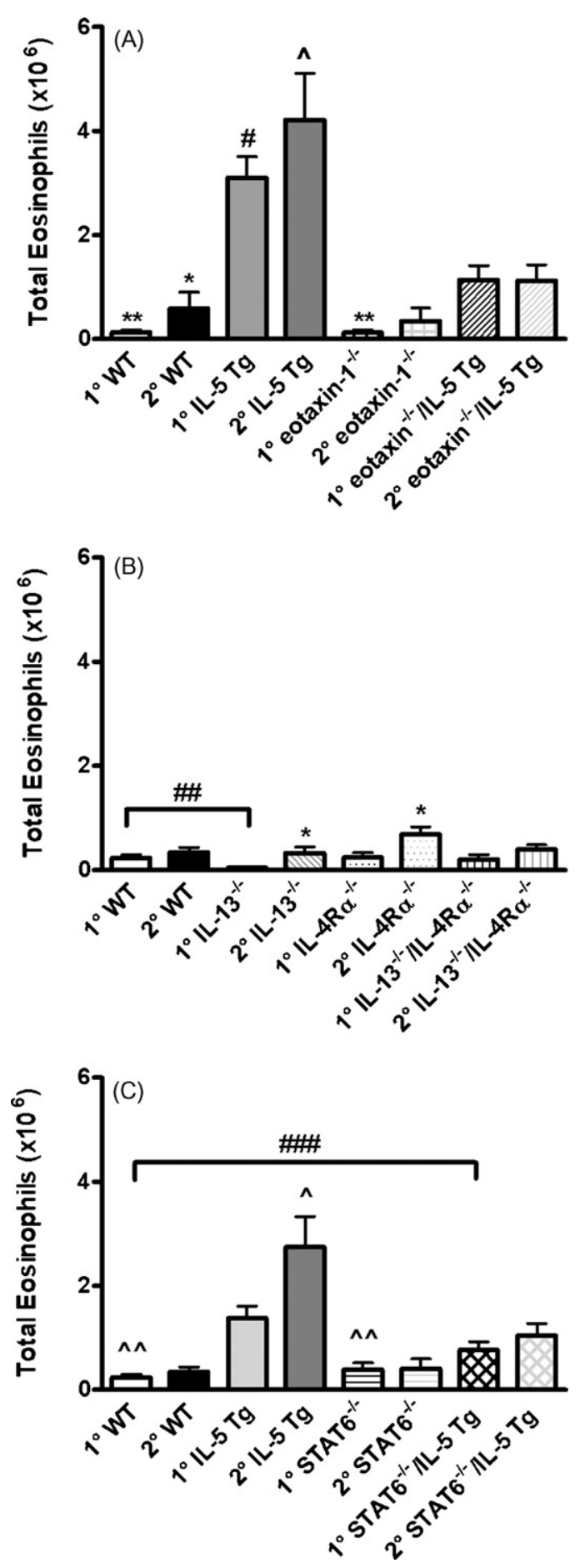

Fig. 1. Eosinophils recovered by lavage of air pouches during primary $\left(1^{\circ}\right)$ and secondary $\left(2^{\circ}\right) N$. brasiliensis infections of WT (A-C), IL-5 Tg (A and C), eotaxin-1 $1^{-1-}$, eotaxin-1-1- /IL-5 Tg (A); IL-13-l-, IL-4R $\alpha^{-1-}$, IL-13 ${ }^{-1-} / \mathrm{IL}-4 \mathrm{R} \alpha^{-1-}$ (B); STAT6 ${ }^{-1-}$ and STAT6 $^{-1-} / \mathrm{IL}-5 \mathrm{Tg}(\mathrm{C})$, mice 4 h p.i. $500 \mathrm{~L} 3 /$ mouse for both $1^{\circ}$ and $2^{\circ}$ infections. Data represent mean eosinophils/pouch lavage + SEM, $n=6-9 /$ group. Significant effect between strains (KW test: for (A) $H=22.81, P=0.0018$; (B) $H=24.6, P=0.0009$; (C) $H=36.55, P<0.0001)$. $^{*}$ : Significantly different to $1^{\circ}$ infection of the same line. \# Significantly different from all other $1^{\circ}$ infection groups. ${ }^{\wedge}$ : Significantly different from all other $2^{\circ}$ infection groups. (A) ${ }^{* *}$ : Significantly different to $1^{\circ}$ eotaxin- $1^{-/-} / \mathrm{IL}-5 \mathrm{Tg}$ group. (B) \#\#: $1^{\circ}$ WT and $1^{\circ}$ IL-13 $3^{-l-}$ significantly different. (C) ${ }^{\varkappa}$ : Significantly different from $1^{\circ}$ IL-5 Tg group. \#\#\#: $1^{\circ}$ WT and $1^{\circ}$ STAT6 ${ }^{-1-} /$ IL-5 Tg groups significantly different (MW post hoc tests, $P<0.05$ ). Data combined from 2 separate experiments. WT groups in (B) and (C) are from the same experiments. 

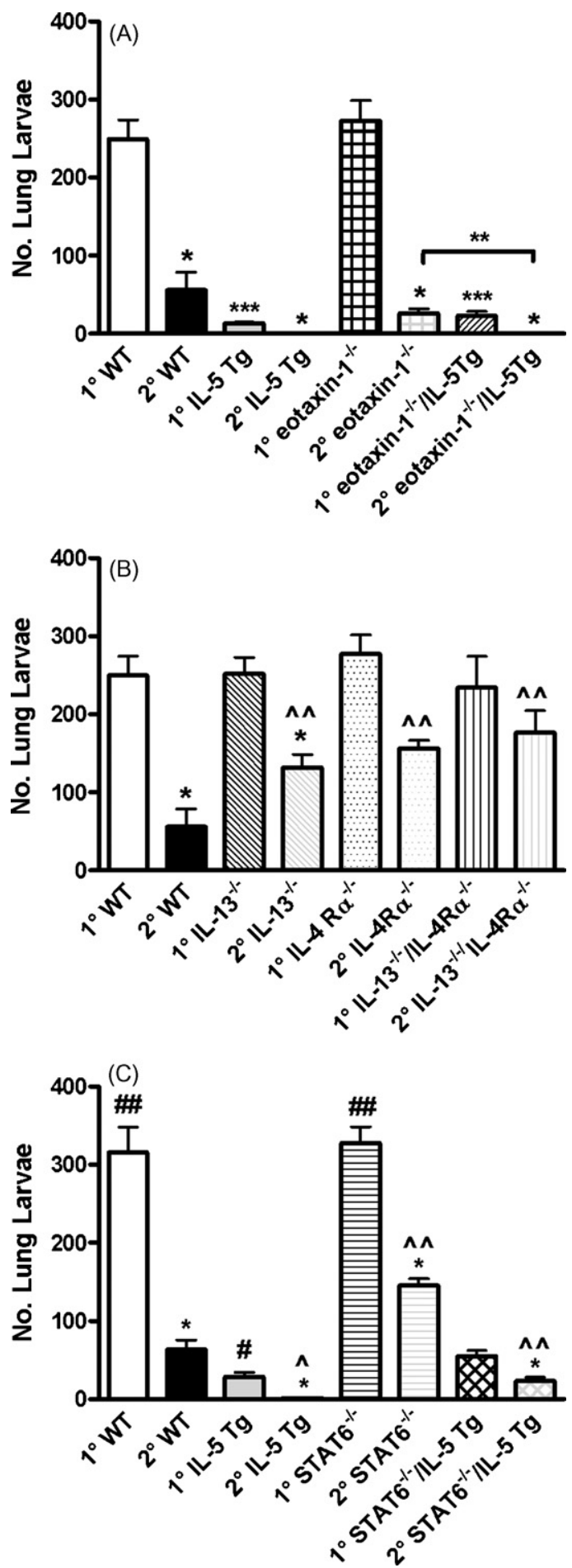

Fig. 2. Recovery of $N$. brasiliensis lung larvae during primary $\left(1^{\circ}\right)$ and secondary $\left(2^{\circ}\right)$ infections of WT (A-C), IL-5 Tg (A and C), eotaxin- $1^{-1-}$ and eotaxin- $1^{-1-} / \mathrm{IL}-5 \mathrm{Tg}(\mathrm{A})$, IL-13 $^{-1-}$, IL-4R $\alpha^{-1-}$, IL-13 ${ }^{-1-} /$ IL-4R $^{-1-}$ (B), and STAT6 ${ }^{-1-}$ and STAT6 ${ }^{-1-} /$ IL-5 Tg (C) mice on day 2 p.i. $750 \mathrm{~L} 3 /$ mouse for both $1^{\circ}$ and $2^{\circ}$ infections. Mean number of lung larvae recovered/mouse + SEM, $n=3-8$ mice/group. Significant effect between strains (KW test (A) $H=27.81, P=0.0002$; (B) $H=19.37, P=0.0071$; (C) $H=48.76$, $P<0.0001)$. *: Significantly different from $1^{\circ}$ infection of the same line. \#: Significantly different from all other $1^{\circ}$ infection groups. ${ }^{* * *}$ : Significantly different from all other $1^{\circ}$ infection groups without an IL-5 transgene. ${ }^{\wedge}$ : Significantly different from all other $2^{\circ}$ infection groups. ${ }^{\varkappa}$ : Significantly different from $2^{\circ}$ WT group. (A) ${ }^{* *}: 2$ eotaxin-1 $1^{-/-}$and $2^{\circ}$ eotaxin-1-/- $/$ IL-5 Tg significantly different. (C) \#\#: Significantly different from $1^{\circ}$ STAT6 $^{-1-} / \mathrm{IL}-5 \mathrm{Tg}$ group (MW post hoc tests, $P<0.05$ ). Data in (A) and (B) from a single experiment and WT data is the same. Data in (C) combined from 2 separate experiments. days 6 to 8 p.i. We assessed egg production on day 6 p.i. in both primary and secondary infections. During primary infections, eggs were detected in all strains not carrying an IL-5 transgene. During primary infections of IL-5 Tg, eotaxin- $1^{-/}-/ \mathrm{IL}-5 \mathrm{Tg}$ and STAT6 ${ }^{-/}-/ \mathrm{IL}-$ $5 \mathrm{Tg}$ animals, few to no eggs were detected (mean $<400$ eggs/gram of faeces compared with mean $+\mathrm{SEM}=7400+4200$ eggs $/$ gram of faeces in WT animals) and none were seen in secondary infections of these IL-5 Tg strains or in WT mice.

Parasite egg production was monitored from days 6 to 27 p.i. during primary and secondary infections of WT, IL-13-l-, IL-4R $\alpha^{-1-}$, IL-13 ${ }^{-1-} /$ IL-4R $\alpha^{-1-}$ and STAT6 ${ }^{-1-}$ mice to assess worm persistence and fecundity. During primary infections, eggs were numerous in all but WT mice until day 10 and slowly declined from day 14 (Fig. 4), with small numbers of eggs recovered until at least days 17-21 in all knockout lines (data not shown). During secondary infections, eggs were not detected in WT mice on any day, however, eggs were found in all knockout lines until at least day 14 p.i. (Fig. 4). In a separate experiment, egg production was monitored in eotaxin$1^{-/-}$mice from days 6 to 8 p.i. of a primary infection. As with WT control animals, no eggs were detected in eotaxin $-1^{-1-}$ mice after day 7 (mean + SEM eggs/gram faeces for WT and eotaxin-1-1groups $4644+2171 /$ mouse and $6926+2289 /$ mouse respectively on day 7).

Worm numbers were evaluated on day 7 p.i. in mice with primary and secondary infections (Fig. 5). Overall, there was a significant effect between strains for primary vs. secondary infections, but when comparing only primary infections, eotaxin-1 $1^{-1-}$, STAT6 $^{-/-}$, IL-13-/-, IL-4R $\alpha^{-/-}$and IL-13-/- /IL-4R $\alpha^{-/-}$animals had similar numbers of worms to WT mice. Also during primary infections, mice carrying the IL-5 transgene, with or without the deletion of eotaxin or STAT6, had markedly reduced worm numbers relative to WT mice. On day 7 of secondary infections, no or few worms were recovered from WT, IL-5 Tg and eotaxin $-1^{-1-}$ mice either with or without the IL- 5 transgene as a second mutation (Fig. 5A). These and other data for eotaxin-1-deficient mice, suggest that this chemokine may not be critical for protection during the intestinal phase of $N$. brasiliensis infections. Interestingly, all other groups tested (STAT6 ${ }^{-1-} \pm \mathrm{IL}-5 \mathrm{Tg}, \mathrm{IL}^{-13^{-/-}}$, IL-4R $\alpha^{-1-}$ and IL-13-/- /IL-4R $\alpha^{-/-}$) had between 15 and 115 worms on day 7 of secondary infection (Fig. 5B and C). Notably, deletion of STAT6 did negatively impact on both primary and secondary resistance conferred by over-expression of IL-5 (Fig. 5C). These data also demonstrate that even during secondary challenge in the absence of the IL-4/IL-13/STAT6 signalling pathway, although worm colonization and/or fecundity are impaired, worm expulsion is also delayed.

We evaluated eosinophil numbers in the small intestine on day 7 p.i. by enumerating eosinophils/villus crypt unit in both primary and secondary infections (Fig. 6). There was a significant effect between groups and as expected in both primary and secondary infections, IL-5 Tg mice had more eosinophils/VCU than all other groups (Fig. 6A and C). While mice deficient in eotaxin-1 did have eosinophils present in the small intestine in both primary and secondary infections, during the latter, numbers were reduced relative to WT mice (Fig. 6A). Mice deficient in eotaxin but also carrying the IL-5 transgene had significantly reduced numbers of eosinophils compared with IL-5 Tg mice with a normal eotaxin gene. STAT6 ${ }^{-1-} / \mathrm{IL}-5 \mathrm{Tg}$ mice also had reduced numbers of eosinophils compared with IL-5 Tg mice (Fig. 6C) and these data suggest that during an intestinal parasitic helminth infection, eosinophil recruitment in the gut is regulated at least in part by eotaxin via a STAT6-dependent mechanism. Other lines of knockout mice without IL-5 transgene expression had similar or slightly less eosinophils than WT animals in both primary and secondary infections, with the greatest negative affect seen in primary IL-4R $\alpha^{-/-}$ mice (Fig. 6B). 


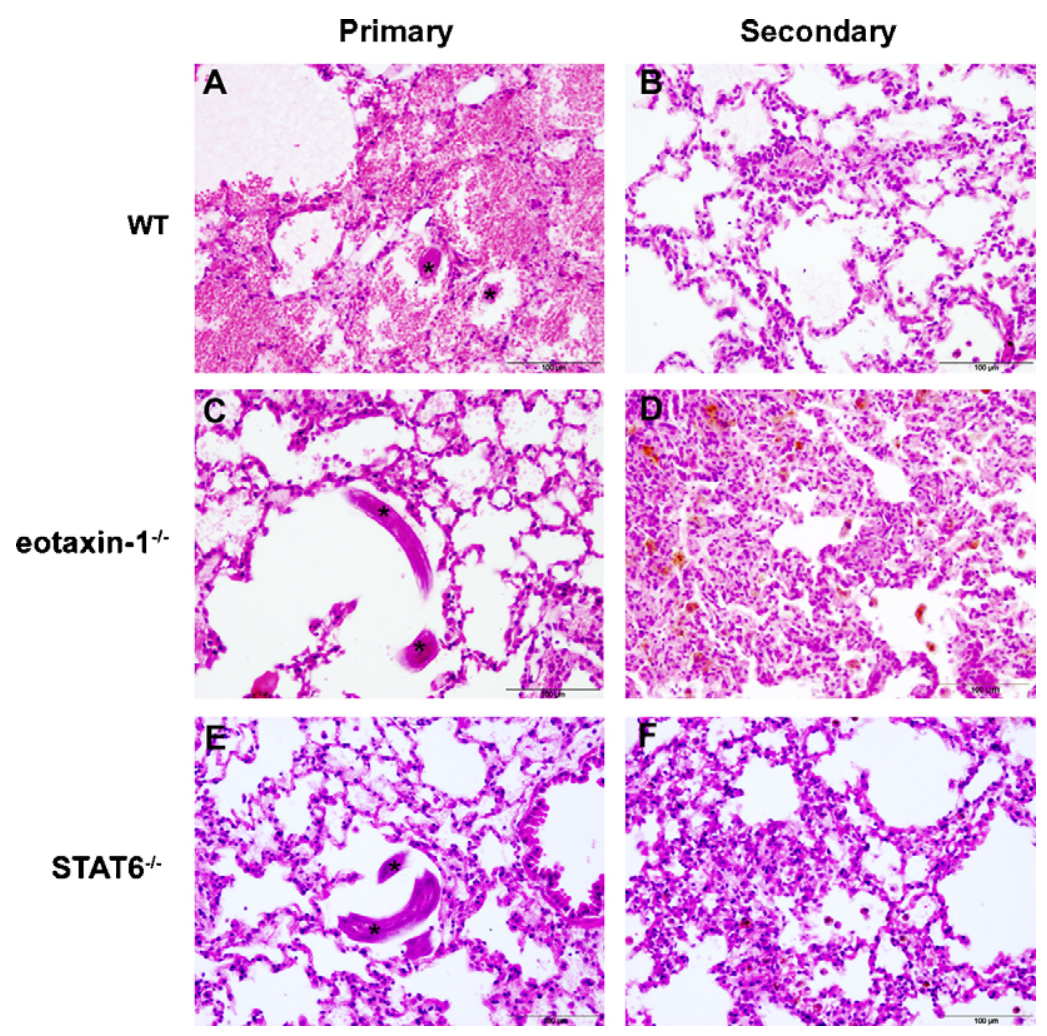

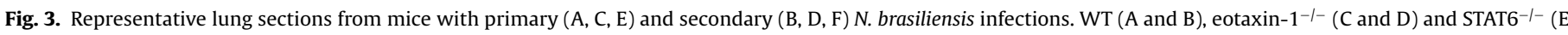
and F) mice. $150 \times$ magnification and scale bars represent $100 \mu \mathrm{m}$. H \& E staining. *: Larvae.



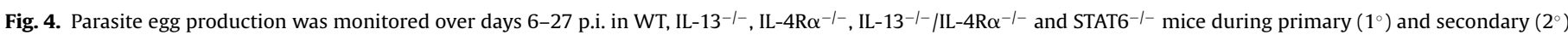

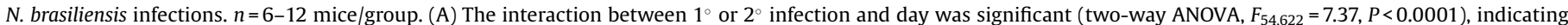

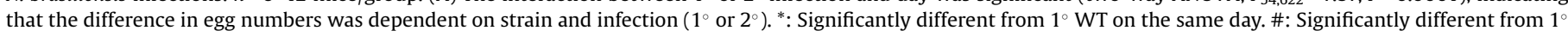
infection of the same line on the same day (Bonferroni post hoc tests, $P<0.05$ ). Data combined from 2 separate experiments. $0=$ none detected.

\subsection{H. bakeri infections}

While it has long been known that some strains of WT mice (e.g. $\mathrm{BALB} / \mathrm{c}, \mathrm{CBA}, \mathrm{A} / \mathrm{J}, \mathrm{C} 3 \mathrm{H}$ and $\mathrm{C} 57 \mathrm{BL} / 6$ ) can become chronically infected with the intestinal nematode $H$. bakeri, the roles of eosinophils and recruitment pathways for these cells have not been investigated. We infected WT, IL-5 Tg, eotaxin-1-/-, eotaxin-1-/- /IL-5 Tg, STAT6 ${ }^{-1-}$ and STAT6 ${ }^{-1}-/ \mathrm{IL}-5 \mathrm{Tg}$ mice with approximately $200 \mathrm{H}$. bakeri L3 and monitored egg production over 116 days (Fig. 7). There were no obvious differences in parasite egg numbers between any of the groups over the time course and egg production was still evident in all strains at the end of the experiment. Worm numbers on day 41 and 117 p.i. were also similar in all groups (data not shown).
These data suggest that eotaxin, eosinophils and the STAT6 signalling pathway do not play a significant role in host protection during primary $H$. bakeri infections.

\section{Discussion}

Eosinophils can be important for early protection during primary and secondary $N$. brasiliensis infections (Daly et al., 1999; Knott et al., 2007; Giacomin et al., 2008). We have previously shown that complement plays a role in the recruitment of eosinophils in the first 30 min of infection, but seems to be less effective by $150 \mathrm{~min}$ (Giacomin et al., 2008). In the current study, we have demonstrated that recruitment of eosinophils to both the skin and small intestine 

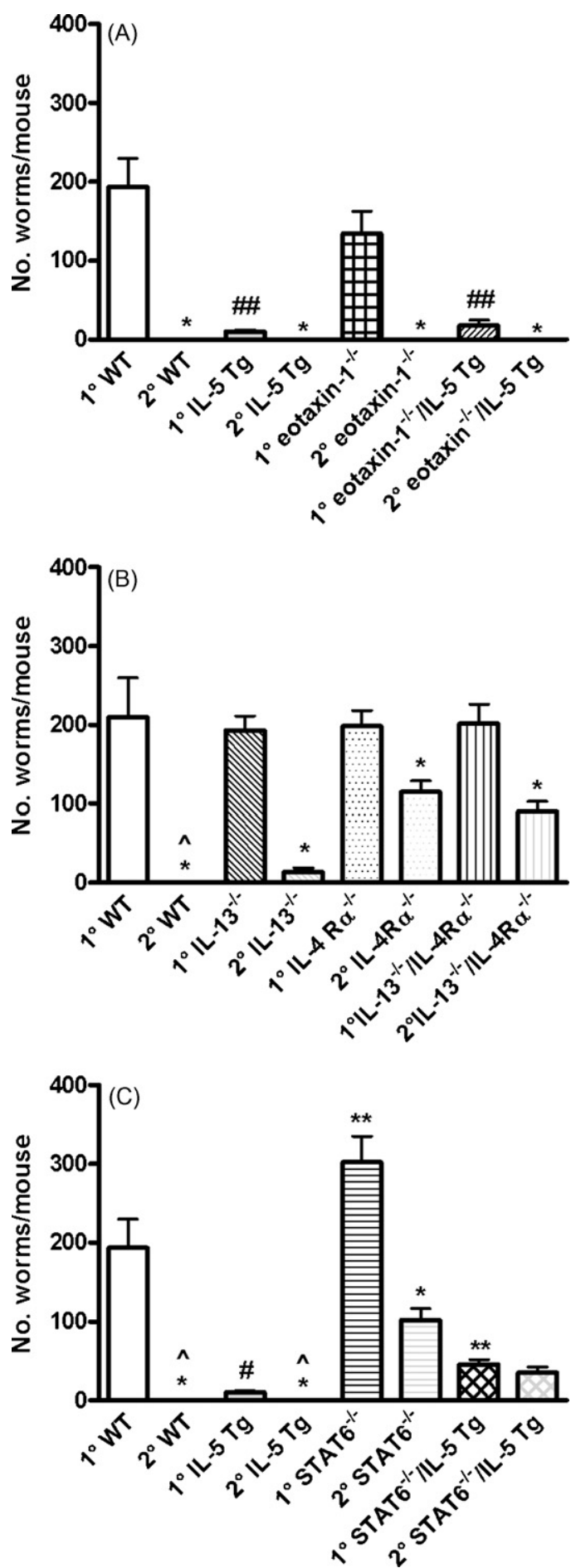

Fig. 5. Day 7 intestinal worm counts (mean worms/mouse + SEM) of WT (A-C), IL-5 Tg (A and C), eotaxin-1 $1^{-1-}$ and eotaxin-1 $1^{-1-} / \mathrm{IL}-5 \mathrm{Tg}$ (A), IL-13-1-, IL-4R $\alpha^{-1-}$, IL-13 ${ }^{-1-} / \mathrm{IL}_{-} 4 \mathrm{R}^{-1-}$ (B), and STAT6 ${ }^{-1-}$ and STAT6 $6^{-/-} / \mathrm{IL}-5 \mathrm{Tg}$ (C) mice. $750 \mathrm{L3} /$ mouse for both $1^{\circ}$ and $2^{\circ}$ infections. $n=3-9$ mice/group. (A-C) Significant effect between strains (KW test (A) $H=45.37, P<0.0001$; (B) $H=26.06, P=0.0005$; (C) $H=49.93$, $P<0.0001)$. $^{*}$ : Significantly different from $1^{\circ}$ infection of the same line. \#: Significantly different from all other $1^{\circ}$ infection groups. \#\#: Significantly different from all other $1^{\circ}$ infection groups without an IL-5 transgene. ${ }^{\wedge}$ : Significantly different from all other $2^{\circ}$ infection groups. $(C)^{* *}$ : Significantly different from $1^{\circ}$ WT group (MW post hoc tests, $P<0.05)$. Data in $(A)$ and $(C)$ combined from 2 separate experiments and WT groups are the same. Data in (B) from a single experiment.
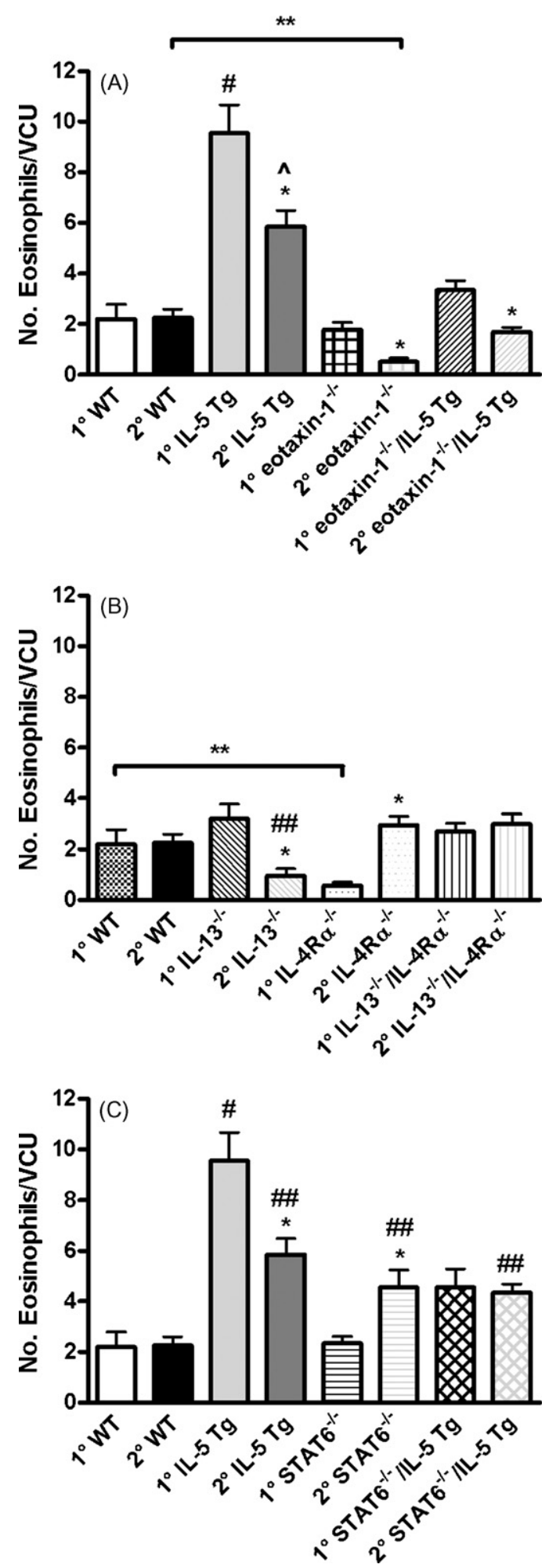

Fig. 6. Mean number of eosinophils/villus crypt unit (VCU) + SEM in sections of small intestine sampled on day 7 p.i. of primary $\left(1^{\circ}\right)$ and secondary $\left(2^{\circ}\right) N$. brasiliensis infections in WT (A-C), IL-5 Tg (A and C), eotaxin- $1^{-1-}$ and eotaxin- $1^{-1-} / \mathrm{IL}-5 \mathrm{Tg}$ (A), IL-13 $^{-1-}$, IL-4R $\alpha^{-/-}$, IL-13 ${ }^{-/-} /$IL-4R $\alpha^{-/-}$(B), STAT6 ${ }^{-1-}$ and STAT6 $6^{-1-} /$ IL-5 Tg (C) mice infected with $750 \mathrm{~L} 3$ for both $1^{\circ}$ and $2^{\circ}$ infections. Eosinophils were counted in 10 villi from each of 2 mice/group. There was a significant effect of strain (KW test (A) $H=55.76, P<0.0001$; (B) $H=33.43, P<0.0001$; (C) $H=45.36, P<0.0001$ ). *: Significantly different from $1^{\circ}$ infection of the same genotype. \#: Significantly different from all $1^{\circ}$ infection groups. ${ }^{\wedge}$ : Significantly different from all $2^{\circ}$ infection groups.\#\#: Significantly different from $2^{\circ}$ WT group. ${ }^{* *}$ : Significant difference between groups, as indicated (MW post hoc tests, $P<0.05$ ). WT (A and C) and IL-5 Tg (A and C) data are the same. 




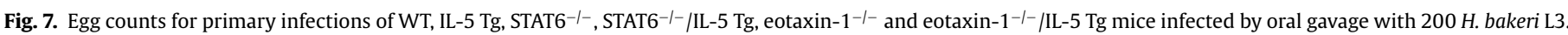
Mean \pm SEM, $n=3$ mice/group.

in response to $N$. brasiliensis infections is impaired in the absence of eotaxin-1. Nevertheless, secondary host resistance in the pre-lung stage of infection is unaffected by eotaxin-1 deficiency and strong primary resistance is also conferred by IL- 5 transgene expression in the absence of this chemokine. This suggests that other mechanisms must compensate in eosinophil recruitment and protection in the absence of eotaxin-1. In contrast, ablation of STAT6, IL-4R $\alpha$ and IL-13 seems to have some impact on primary resistance in both the prelung and intestinal phases of infection, with more larvae migrating to the lungs and prolonged parasite egg production in animals lacking these gene products. STAT6 deficiency was also deleterious in the IL-5 Tg background, though the effect was relatively modest. An absence of these factors during secondary $N$. brasiliensis infections resulted in a reduction in pre-lung and in some instances, intestinal resistance. In contrast, the kinetics of $H$. bakeri infections were unaltered by IL-5 $\mathrm{Tg}$ expression or deletion of eotaxin or STAT6, suggesting that these elements of the immune response contribute little to controlling this intestinal parasite.

IL-4, IL-13, STAT6 and IL-5 play major roles in the pathogenesis of asthma and allergies (Wardlaw and Kay, 1987; Foster et al., 1996; Dent et al., 1997b; Dent, 2002; Zimmermann et al., 2003). IL-4 and IL-13 promote mucus hypersecretion in asthmatic lungs ( $\mathrm{Li}$ et al., 1999; Webb et al., 2000) and may also contribute to airways hyper reactivity and remodelling (Webb et al., 2000). IL-4, the IL-4R $\alpha$ and STAT6 are crucial for the expulsion of adult $N$. brasiliensis worms from the small intestine and an absence of these factors results in prolonged primary infections (Urban et al., 1998; Fallon et al., 2002, 2006). STAT6 is also crucial for expulsion of adult Trichinella spiralis worms from the small intestine (Khan et al., 2001). IL-5, IL-9 and IL-25 may make additional important contributions to expulsion of $N$. brasiliensis from the gut during primary infections (Khan et al., 2001; Fallon et al., 2002, 2006). Sakamoto et al. (2004) have shown impaired resistance in mice deficient in STAT6 during secondary infections. In the latter study, eggs were detected in C57BL/6 STAT6 ${ }^{-1-}$ animals until day 21 post secondary infection, whereas we detected egg production for 17 days p.i. in BALB/c STAT6 ${ }^{-1-}$ animals. In both instances, in the absence of STAT6, worm retention and egg output were prolonged in primary and secondary infections. In this study we have also now shown for the first time that deletion of IL-13 and/or the IL-4R $\alpha$ also results in persistence of worms and production of eggs during secondary infections.

While others have interpreted the impact of STAT6 and IL-4 deletions to be at the level of the gut, our data suggest that deficiency also impacts much earlier, during the pre-lung phase. Damage inflicted at this stage may limit later colonization of the gut. Most importantly, during secondary infections, more lung larvae were detected in IL-13-, IL-4R $\alpha$ - or STAT6-deficient animals than in WT animals. Our data suggest that in secondary infections, an absence of any of these factors facilitated migration of significant numbers of larvae to the lungs and this outcome was not due to poor recruitment of eosinophils to the skin.

In humans, three members of the eotaxin family (eotaxin-1, -2 and -3 ) have been described, whilst in the mouse only eotaxin- 1 and -2 have been reported (reviewed in (Rothenberg and Hogan, 2006)). Eotaxins are highly specific for the recruitment of eosinophils, and the chemokine receptor for the eotaxins is CCR-3 (Kitaura et al., 1996). Eosinophils are recruited into the skin by injection of guinea pig eotaxin (Jose et al., 1994). Eotaxin-1 is expressed at high basal levels throughout the length of the intestine as well as the heart, kidney and pancreas, and at lower levels in the liver, lungs and ovaries (Garcia-Zepeda et al., 1996; Ponath et al., 1996). Eotaxin-2 is primarily expressed in the jejunum and spleen (Zimmermann et al., 2000). Mice deficient in eotaxin-1 as well as mice treated with a neutralizing monoclonal antibody show a reduction in the number of eosinophils in the lungs after induction of allergy (Rothenberg et al., 1997; Gonzalo et al., 1998; Matthews et al., 1998). Mice with a deletion of the CCR3 gene, but not eotaxin-2-deficient mice, have fewer eosinophils in the gastrointestinal tract (Humbles et al., 2002; Pope et al., 2005). The expression of eotaxin is upregulated in the airways of mice over-expressing IL-4 in the lungs and also following intranasal administration of recombinant IL-4, and this is dependent on STAT6 signalling (Zimmermann et al., 2000). Eotaxin levels are increased in the blood (Hossny et al., 2001) and in skin lesions in atopic dermatitis in humans (Yawalkar et al., 1999). In a mouse model of atopic dermatitis, CCR-3 was found to be essential for eosinophil recruitment into the skin (Ma et al., 2002) and eotaxin was detected in skin lesions (Oshio et al., 2009). Therefore we might expect a reduction in eosinophil recruitment to skin and the small intestine in eotaxin-1 ${ }^{-1-}$ mice infected with intestinal helminths.

Although eosinophil recruitment to the skin was similar in WT and eotaxin-1 ${ }^{-/-}$mice in both primary and secondary infections, deletion of eotaxin-1 did reduce eosinophil recruitment into infected skin in IL-5 Tg mice. Nevertheless, the eosinophils that were recruited or other factors that are unique to IL-5 $\mathrm{Tg}$ mice would appear to have been sufficient to provide potent protection, as few parasites were seen in the lungs of eotaxin-1-/- IL-5 $\mathrm{Tg}$ animals during primary or secondary infections.

In primary infections with $N$. brasiliensis, IL-5-deficient animals have significantly higher egg and worm burdens than WT mice (Knott et al., 2007), suggesting that eosinophils in the gut 
may make some contribution to worm expulsion. In the present study we have shown that there were less eosinophils/VCU in the small intestines of eotaxin-1 $1^{-1-}$ than in WT mice on day 7 p.i. of secondary infections, but surprisingly, no difference was detected during primary infections. While eotaxin-1-/-/IL-5 Tg animals had more eosinophils present in small intestines than WT mice during primary infections, numbers were significantly less than in IL-5 Tg mice, suggesting that recruitment in this context was at least partially dependent on eotaxin- 1 . In eotaxin $-1^{-1-}$ mice, parasite eggs were not detected past day 8 in primary infections and during secondary infections, few intestinal worms were detected. Where few larvae successfully migrate to or colonise the intestine, there are likely to be less of the inflammatory stimuli that might contribute to the recruitment of eosinophils. This is the most likely explanation for the relatively low intestinal eosinophil numbers seen during secondary infections in eotaxin-1 ${ }^{-1-}$, WT and IL-5 Tg mice. So while eotaxin-1 does contribute to the recruitment of eosinophils into $N$. brasiliensis-infected gut, any role that eosinophils might play in worm expulsion, is not dependent on this chemokine.

Our data also indicate that alternative eosinophil recruitment pathways can partially compensate for the absence of eotaxin-1. Although eotaxin-1 is a specific recruitment factor for eosinophils, other molecules can also induce the recruitment of these cells in vitro and/or in vivo, including eotaxin-2 (Zimmermann et al., 2000), platelet activating factor (PAF) (Wardlaw et al., 1986), the complement proteins C3a and C5a (Daffern et al., 1995; DiScipio et al., 1999; Giacomin et al., 2008), macrophage inflammatory protein (MIP)- $1 \alpha$, regulated upon activation in normal T cells expressed and secreted (RANTES) (Alam et al., 1993) and monocyte chemo-attractant proteins (MCP)-2 and -3 (Noso et al., 1994). It is likely that one or more of these factors can assist the recruitment of eosinophils and other leukocytes into the skin during secondary $N$. brasiliensis infections of eotaxin-1-1- mice. Disruption of the CCR-3 gene dramatically reduces jejunal eosinophilia in response to $T$. spiralis infections, but some eosinophils are detected within the caecum in infected animals, suggesting that alternative mechanism/s can compensate in recruitment in the absence of eotaxin and other chemokine ligands of this receptor (Gurish et al., 2002). Future investigations of eosinophil recruitment in response to intestinal nematodes should address the presence of alternative and compensatory recruitment factors within the first few hours of exposure, as well as in the gut phase of infection.

When WT, IL-5 Tg, eotaxin-1-1- \pm IL-5 Tg and STAT6 ${ }^{-1-} \pm$ IL-5 Tg mice were infected with $H$. bakeri, an intestinal nematode which can reside in hosts for months during a primary infection, we saw no obvious differences in faecal egg numbers over 116 days of primary infection. Therefore over-expression of IL-5, which confers strong resistance to N. brasiliensis (Daly et al., 1999; Dent et al., 1999; Knott et al., 2007 and this study), does not protect against $H$. bakeri. In this longer term parasite model, eotaxin-1 and the STAT6 signalling pathway also do not play important roles in host protection. This is most likely due to the different lifecycles of the two parasites, with $N$. brasiliensis migrating through the skin, lungs and small intestine, whereas $H$. bakeri spends its life only in the small intestine. Importantly, H. bakeri is also immunosuppressive (Pritchard et al., 1984; Pritchard and Behnke, 1985; Crawford et al., 1989) and in this context, eotaxin and STAT6 may have little potential to contribute to parasite expulsion.

This study contributes to the characterisation of the cells, cytokines and chemokines that are responsible for protection in the early pre-lung stage of $N$. brasiliensis infections. Our data suggest that eotaxin-1 does not play an essential role in protection in the pre-lung or intestinal phases of primary or secondary infections. However, our most novel and important finding is that IL-4, IL-13 and STAT6 not only play important roles in adult worm expulsion, but also in protection during the early pre-intestinal stages of secondary infections. Current treatments for intestinal helminth infections often target adult worms, but we have now shown that at least for parasite species that enter the host through the skin, the pre-lung stage might prove to be a more useful focus for vaccines and/or development of new treatment strategies.

\section{Acknowledgements}

This work was funded by the National Health and Medical Research Council of Australia through a project grant and University of Adelaide support (L.A.D.) and a National Health and Medical Research Council Program grant (K.I.M. and P.S.F.). We thank Drs. Mariela Segura, Zhong Su and Mary Stevenson (McGill University, Montreal, Canada) for providing the initial source of $H$. bakeri.

\section{References}

Alam, R., Stafford, S., Forsythe, P., Harrison, R., Faubion, D., Lett-Brown, M.A., Grant J.A., 1993. RANTES is a chemotactic and activating factor for human eosinophils. J. Immunol. 150, 3442-3448.

Cable, J., Harris, P.D., Lewis, J.W., Behnke, J.M., 2006. Molecular evidence that Heligmosomoides polygyrus from laboratory mice and wood mice are separate species. Parasitology 133, 111-122.

Collins, P.D., Marleau, S., Griffiths-Johnson, D.A., Jose, P.J., Williams, T.J., 1995. Cooperation between interleukin- 5 and the chemokine eotaxin to induce eosinophil accumulation in vivo. J. Exp. Med. 182, 1169-1174.

Crawford, C., Behnke, J.M., Pritchard, D.I., 1989. Suppression of heterologous immunity by Nematospiroides dubius antigens in vitro. Int. J. Parasitol. 19, 29-34.

Daffern, P.J., Pfeifer, P.H., Ember, J.A., Hugli, T.E., 1995. C3a is a chemotaxin for human eosinophils but not for neutrophils. I. C3a stimulation of neutrophils is secondary to eosinophil activation. J. Exp. Med. 181, 2119-2127.

Daly, C.M., 1999. Immune responses to Nippostrongylus brasiliensis in interleukin-5 transgenic mice. In: Microbiology and Immunology. The University of Adelaide, Adelaide, p. 208

Daly, C.M., Mayrhofer, G., Dent, L.A., 1999. Trapping and immobilization of Nippostrongylus brasiliensis larvae at the site of inoculation in primary infections of interleukin-5 transgenic mice. Infect. Immun. 67, 5315-5323.

Dent, L.A., 2002. For better or worse: common determinants influencing health and disease in parasitic infections, asthma and reproductive biology. J. Reprod. Immunol. 57, 255

Dent, L.A., Daly, C., Geddes, A., Cormie, J., Finlay, D.A., Bignold, L., Hagan, P., Parkhouse, R.M., Garate, T., Parsons, J., Mayrhofer, G., 1997b. Immune responses of IL-5 transgenic mice to parasites and aeroallergens. Mem. Inst. Oswaldo Cruz 92, 45-54.

Dent, L.A., Daly, C.M., Mayrhofer, G., Zimmerman, T., Hallett, A., Bignold, L.P., Creaney, J., Parsons, J.C., 1999. Interleukin-5 transgenic mice show enhanced resistance to primary infections with Nippostrongylus brasiliensis but not primary infections with Toxocara canis. Infect. Immun. 67, 989-993.

Dent, L.A., Strath, M., Mellor, A.L., Sanderson, C.J., 1990. Eosinophilia in transgenic mice expressing interleukin 5. J. Exp. Med. 172, 1425-1431.

DiScipio, R.G., Daffern, P.J., Jagels, M.A., Broide, D.H., Sriramarao, P., 1999. A comparison of C3a and C5a-mediated stable adhesion of rolling eosinophils in postcapillary venules and transendothelial migration in vitro and in vivo. J. Immunol. 162, 1127-1136.

Fallon, P.G., Ballantyne, S.J., Mangan, N.E., Barlow, J.L., Dasvarma, A., Hewett, D.R., McIlgorm, A., Jolin, H.E., McKenzie, A.N., 2006. Identification of an interleukin (IL)-25-dependent cell population that provides IL-4, IL-5, and IL-13 at the onset of helminth expulsion. J. Exp. Med. 203, 1105-1116.

Fallon, P.G., Jolin, H.E., Smith, P., Emson, C.L., Townsend, M.J., Fallon, R., McKenzie, A.N., 2002. IL-4 induces characteristic Th2 responses even in the combined absence of IL-5, IL-9, and IL-13. Immunity 17, 7-17.

Foster, P.S., Hogan, S.P., Ramsay, A.J., Matthaei, K.I., Young, I.G., 1996. Interleukin 5 deficiency abolishes eosinophilia, airways hyperreactivity, and lung damage in a mouse asthma model. J. Exp. Med. 183, 195-201.

Garcia-Zepeda, E.A., Rothenberg, M.E., Ownbey, R.T., Celestin, J., Leder, P., Luster A.D., 1996. Human eotaxin is a specific chemoattractant for eosinophil cells and provides a new mechanism to explain tissue eosinophilia. Nat. Med. 2, 449-456.

Giacomin, P.R., Gordon, D.L., Botto, M., Daha, M.R., Sanderson, S.D., Taylor, S.M., Dent, L.A., 2008. The role of complement in innate, adaptive and eosinophil-dependent immunity to the nematode Nippostrongylus brasiliensis. Mol. Immunol. 45, 446-455.

Giacomin, P.R., Wang, H., Gordon, D.L., Botto, M., Dent, L.A., 2005. Loss of complement activation and leukocyte adherence as Nippostrongylus brasiliensis develops within the murine host. Infect. Immun. 73, 7442-7449.

Gonzalo, J.A., Lloyd, C.M., Kremer, L., Finger, E., Martinez, A.C., Siegelman, M.H., Cybulsky, M., Gutierrez-Ramos, J.C., 1996. Eosinophil recruitment to the lung in a murine model of allergic inflammation. The role of T cells, chemokines, and adhesion receptors. J. Clin. Invest. 98, 2332-2345.

Gonzalo, J.A., Lloyd, C.M., Wen, D., Albar, J.P., Wells, T.N., Proudfoot, A., Martinez, A.C., Dorf, M., Bjerke, T., Coyle, A.J., Gutierrez-Ramos, J.C., 1998. The coordinated action 
of CC chemokines in the lung orchestrates allergic inflammation and airway hyperresponsiveness. J. Exp. Med. 188, 157-167.

Gurish, M.F., Humbles, A., Tao, H., Finkelstein, S., Boyce, J.A., Gerard, C., Friend, D.S., Austen, K.F., 2002. CCR3 is required for tissue eosinophilia and larval cytotoxicity after infection with Trichinella spiralis. J. Immunol. 168, 5730-5736.

Hogan, S.P., Mishra, A., Brandt, E.B., Foster, P.S., Rothenberg, M.E., 2000. A critical role for eotaxin in experimental oral antigen-induced eosinophilic gastrointestinal allergy. Proc. Natl. Acad. Sci. U.S.A. 97, 6681-6686.

Hossny, E., Aboul-Magd, M., Bakr, S., 2001. Increased plasma eotaxin in atopic dermatitis and acute urticaria in infants and children. Allergy 56, 996-1002.

Humbles, A.A., Lu, B., Friend, D.S., Okinaga, S., Lora, J., Al-Garawi, A., Martin, T.R. Gerard, N.P., Gerard, C., 2002. The murine CCR3 receptor regulates both the role of eosinophils and mast cells in allergen-induced airway inflammation and hyperresponsiveness. Proc. Natl. Acad. Sci. U.S.A. 99, 1479-1484.

Jose, P.J., Griffiths-Johnson, D.A., Collins, P.D., Walsh, D.T., Moqbel, R., Totty, N.F., Truong, O., Hsuan, J.J., Williams, T.J., 1994. Eotaxin: a potent eosinophil chemoattractant cytokine detected in a guinea pig model of allergic airways inflammation. J. Exp. Med. 179, 881-887.

Kaplan, M.H., Schindler, U., Smiley, S.T., Grusby, M.J., 1996. Stat6 is required for mediating responses to IL-4 and for development of Th2 cells. Immunity 4, 313-319.

Khan, W.I., Vallance, B.A., Blennerhassett, P.A., Deng, Y., Verdu, E.F., Matthaei, K.I., Collins, S.M., 2001. Critical role for signal transducer and activator of transcription factor 6 in mediating intestinal muscle hypercontractility and worm expulsion in Trichinella spiralis-infected mice. Infect. Immun. 69, 838-844.

Kitaura, M., Nakajima, T., Imai, T., Harada, S., Combadiere, C., Tiffany, H.L., Murphy, P.M., Yoshie, O., 1996. Molecular cloning of human eotaxin, an eosinophilselective CC chemokine, and identification of a specific eosinophil eotaxin receptor, CC chemokine receptor 3. J. Biol. Chem. 271, 7725-7730.

Knott, M.L., Matthaei, K.I., Giacomin, P.R., Wang, H., Foster, P.S., Dent, L.A., 2007. Impaired resistance in early secondary Nippostrongylus brasiliensis infections in mice with defective eosinophilopoeisis. Int. J. Parasitol. 37, 1367-1378.

Kweon, M.N., Yamamoto, M., Kajiki, M., Takahashi, I., Kiyono, H., 2000. Systemically derived large intestinal CD4(+) Th2 cells play a central role in STAT6-mediated allergic diarrhea. J. Clin. Invest. 106, 199-206.

Lawrence, R.A., Gray, C.A., Osborne, J., Maizels, R.M., 1996. Nippostrongylus brasiliensis: cytokine responses and nematode expulsion in normal and IL-4-deficient mice. Exp. Parasitol. 84, 65-73.

Li, L., Xia, Y., Nguyen, A., Lai, Y.H., Feng, L., Mosmann, T.R., Lo, D., 1999. Effects of Th2 cytokines on chemokine expression in the lung: IL-13 potently induces eotaxin expression by airway epithelial cells. J. Immunol. 162, 2477-2487.

Ma, W., Bryce, P.J., Humbles, A.A., Laouini, D., Yalcindag, A., Alenius, H., Friend, D.S., Oettgen, H.C., Gerard, C., Geha, R.S., 2002. CCR3 is essential for skin eosinophilia and airway hyperresponsiveness in a murine model of allergic skin inflammation. J. Clin. Invest. 109, 621-628.

Madden, K.B., Whitman, L., Sullivan, C., Gause, W.C., Urban Jr., J.F., Katona, I.M., Finkelman, F.D., Shea-Donohue, T., 2002. Role of STAT6 and mast cells in IL4- and IL-13-induced alterations in murine intestinal epithelial cell function. J. Immunol. 169, 4417-4422.

Madden, K.B., Yeung, K.A., Zhao, A., Gause, W.C., Finkelman, F.D., Katona, I.M., Urban Jr., J.F., Shea-Donohue, T., 2004. Enteric nematodes induce stereotypic STAT6dependent alterations in intestinal epithelial cell function. J. Immunol. 172, 5616-5621.

Matthews, A.N., Friend, D.S., Zimmermann, N., Sarafi, M.N., Luster, A.D., Pearlman, E., Wert, S.E., Rothenberg, M.E., 1998. Eotaxin is required for the baseline level of tissue eosinophils. Proc. Natl. Acad. Sci. U.S.A. 95, 6273-6278.

Mattoli, S., Stacey, M.A., Sun, G., Bellini, A., Marini, M., 1997. Eotaxin expression and eosinophilic inflammation in asthma. Biochem. Biophys. Res. Commun. 236, 299-301.

McKenzie, G.J., Emson, C.L., Bell, S.E., Anderson, S., Fallon, P., Zurawski, G., Murray, R., Grencis, R., McKenzie, A.N., 1998. Impaired development of Th2 cells in IL-13deficient mice. Immunity 9, 423-432.

Mishra, A., Hogan, S.P., Lee, J.J., Foster, P.S., Rothenberg, M.E., 1999. Fundamental signals that regulate eosinophil homing to the gastrointestinal tract. J. Clin. Invest. 103, 1719-1727.

Mochizuki, M., Bartels, J., Mallet, A.I., Christophers, E., Schroder, J.M., 1998. IL-4 induces eotaxin: a possible mechanism of selective eosinophil recruitment in helminth infection and atopy. J. Immunol. 160, 60-68.

Moore, P.E., Church, T.L., Chism, D.D., Panettieri Jr., R.A., Shore, S.A., 2002. IL-13 and IL-4 cause eotaxin release in human airway smooth muscle cells: a role for ERK. Am. J. Physiol. Lung Cell Mol. Physiol. 282, L847-L853.
Mould, A.W., Matthaei, K.I., Young, I.G., Foster, P.S., 1997. Relationship between interleukin-5 and eotaxin in regulating blood and tissue eosinophilia in mice. J. Clin. Invest. 99, 1064-1071.

Noben-Trauth, N., Shultz, L.D., Brombacher, F., Urban Jr., J.F., Gu, H., Paul, W.E. 1997. An interleukin 4 (IL-4)-independent pathway for CD4 + T cell IL-4 production is revealed in IL-4 receptor-deficient mice. Proc. Natl. Acad. Sci. U.S.A. 94 10838-10843.

Noso, N., Proost, P., Van Damme, J., Schroder, J.M., 1994. Human monocyte chemotactic proteins-2 and 3 (MCP-2 and MCP-3) attract human eosinophils and desensitize the chemotactic responses towards RANTES. Biochem. Biophys. Res. Commun. 200, 1470-1476.

Oshio, T., Sasaki, Y., Funakoshi-Tago, M., Aizu-Yokota, E., Sonoda, Y., Matsuoka, H., Kasahara, T., 2009. Dermatophagoides farinae extract induces severe atopic dermatitis in $\mathrm{NC} / \mathrm{Nga}$ mice, which is effectively suppressed by the administration of tacrolimus ointment. Int. Immunopharmacol. 9, 403-411.

Ponath, P.D., Qin, S., Ringler, D.J., Clark-Lewis, I., Wang, J., Kassam, N., Smith, H., Shi, X., Gonzalo, J.A., Newman, W., Gutierrez-Ramos, J.C., Mackay, C.R., 1996. Cloning of the human eosinophil chemoattractant, eotaxin. Expression, receptor binding, and functional properties suggest a mechanism for the selective recruitment of eosinophils. J. Clin. Invest. 97, 604-612.

Pope, S.M., Fulkerson, P.C., Blanchard, C., Akei, H.S., Nikolaidis, N.M., Zimmermann, N., Molkentin, J.D., Rothenberg, M.E., 2005. Identification of a cooperative mechanism involving interleukin-13 and eotaxin-2 in experimental allergic lung inflammation. J. Biol. Chem. 280, 13952-13961.

Pritchard, D.I., Ali, N.M., Behnke, J.M., 1984. Analysis of the mechanism of immunodepression following heterologous antigenic stimulation during concurrent infection with Nematospiroides dubius. Immunology 51, 633642.

Pritchard, D.I., Behnke, J.M., 1985. The suppression of homologous immunity by soluble adult antigens of Nematospiroides dubius. J. Helminthol. 59, 251-256.

Rothenberg, M.E., Hogan, S.P., 2006. The eosinophil. Annu. Rev. Immunol. 24, 147-174.

Rothenberg, M.E., MacLean, J.A., Pearlman, E., Luster, A.D., Leder, P., 1997. Targeted disruption of the chemokine eotaxin partially reduces antigen-induced tissue eosinophilia. J. Exp. Med. 185, 785-790.

Sakamoto, Y., Hiromatsu, K., Ishiwata, K., Inagaki-Ohara, K., Ikeda, T., NakamuraUchiyama, F., Nawa, Y., 2004. Chronic intestinal nematode infection induces Stat6-independent interleukin-5 production and causes eosinophilic inflammatory responses in mice. Immunology 112, 615-623.

Sheridan, J.W., Finlay-Jones, J.J., 1977. Studies on a fractionated murine fibrosarcoma: a reproducible method for the cautious and a caution for the unwary. J. Cell Physiol. 90, 535-552.

Urban Jr., J.F., Noben-Trauth, N., Donaldson, D.D., Madden, K.B., Morris, S.C., Collins, M., Finkelman, F.D., 1998. IL-13, IL-4Ralpha, and Stat6 are required for the expulsion of the gastrointestinal nematode parasite Nippostrongylus brasiliensis. Immunity 8, 255-264.

Urban Jr., J.F., Noben-Trauth, N., Schopf, L., Madden, K.B., Finkelman, F.D., 2001 Cutting edge: IL-4 receptor expression by non-bone marrow-derived cells is required to expel gastrointestinal nematode parasites. J. Immunol. 167, 6078-6081.

Wardlaw, A.J., Kay, A.B., 1987. The role of the eosinophil in the pathogenesis of asthma. Allergy 42, 321-335.

Wardlaw, A.J., Moqbel, R., Cromwell, O., Kay, A.B., 1986. Platelet-activating factor A potent chemotactic and chemokinetic factor for human eosinophils. J. Clin. Invest. 78, 1701-1706.

Webb, D.C., McKenzie, A.N., Koskinen, A.M., Yang, M., Mattes, J., Foster, P.S., 2000 Integrated signals between IL-13, IL-4, and IL-5 regulate airways hyperreactivity. J. Immunol. 165, 108-113.

Yawalkar, N., Uguccioni, M., Scharer, J., Braunwalder, J., Karlen, S., Dewald, B., Braathen, L.R., Baggiolini, M., 1999. Enhanced expression of eotaxin and CCR3 in atopic dermatitis. J. Invest. Dermatol. 113, 43-48.

Zimmermann, N., Hershey, G.K., Foster, P.S., Rothenberg, M.E., 2003. Chemokines in asthma: cooperative interaction between chemokines and IL-13. J. Allergy Clin. Immunol. 111, 227-242 (quiz 243).

Zimmermann, N., Hogan, S.P., Mishra, A., Brandt, E.B., Bodette, T.R., Pope, S.M., Finkelman, F.D., Rothenberg, M.E., 2000. Murine eotaxin-2: a constitutive eosinophil chemokine induced by allergen challenge and IL-4 overexpression. J. Immunol. $165,5839-5846$. 